\title{
QUANTUM OCCASIONALISM
}

\author{
Vasil Penchev, vasildinev@gmail.com \\ Bulgarian Academy of Sciences: \\ Institute of Philosophy and Sociology: \\ Dept. of Logical Systems and Models
}

\begin{abstract}
Both transition and transformation link the ideal and material into a whole. Future is what "causes" the present, and the latter in turn is what "causes" the past. That kind of "reverse causality" needs free choice and free will in the present in order to be able to be realized unlike classical causality. A few properties feature the concept of "quantum occasionalism" as follows. Some hypothetical entity generates successively a series of well-ordered states. That hypothetical entity is called "coherent state" in quantum mechanics and defined as a superposition of all possible states of the quantum system. The already generated well-ordered series can be interpreted as a causal sequence. Thus the generating cause remains hidden behind the visible well-ordering of the series and hides itself behind the perfect visible order created by it. That visible order only seems to cause itself by itself.
\end{abstract}

KEY WORDS: causality, choice, freewill, occasionalism, quantum occasionalism, reverse causality

\section{NTRODUCTION}

The thesis is: Indeterminism in quantum mechanics can be interpreted as a form of occasionalism, which is called "quantum occasionalism". It in turn implies the option for any probabilistic determinism and thus for causal determinism as a particular case to be extended equivalently to that form of occasionalism.

Two necessary comments of the thesis:

1. The meant form of occasionalilsm can be formulated as follows: Some hypothetical entity generates successively a series of well-ordered states. That hypothetical entity is called "coherent state" in quantum mechanics and defined as a superposition of all possible states of the quantum system, which is under consideration. The already generated well-ordered series of states admits, but does not requires, to be interpreted as a causal sequence since the causal sequence is isomorphic to the already generated well-ordered series and therefor can replace it. Thus the generating cause, namely the hypothetical entity in question or the coherent state in quantum mechanics, remains hidden behind the visible well-ordering of the series. In fact, the generating cause hides itself behind the perfect visible order created by it. That visible order only seems to cause itself by itself.

2. The same form of occasionalism can be interpreted in turn in terms of the inverse of time, i.e. from future via the present to the past, as follows: Future generates successively a wellordered series of states in the past by the meditation of the present. The essence of the present is some series of choices, which orders the absolutely unordered future into the well-ordered 
sequences of events in the past. That "inverted" time from future to the past hides itself behind the usual "arrow of time" from the past to the future therefor implying a secondary order of causality in this hiding direction of time, however accepted as the real course of time according to common sense.

A few main arguments for the thesis:

1. The theorems about the absence of hidden variables in quantum mechanics (Neumann 1932; Kochen and Specker 1968) exclude any well-ordering in any coherent state before measurement. Nevertheless, the same state turns out to be well-ordered after measurement, e.g. by the parameter of registration time, therefore constituting an equivalent statistical ensemble. The relation between the states before and after measurement requires necessarily the wellordering theorem equivalent to the axiom of choice.

2. Consequently, any coherent state generates an equivalent statistical ensemble of measured results already well-ordered in the past. However, any result from this set of measured results does not cause any other results for all results are fundamentally random, i.e. occasional. Each of them originates from the coherent state immediately rather than from any other result preceding it. Thus quantum mechanics allows of seeing a common really generating cause hidden until now: The usual causal order is only apparent in fact and even can be absent as in the case investigated by quantum mechanics.

3. Nevertheless, the apparent causal order can be restored even as to quantum mechanics in relation the sequences of coherent states or equivalent statistical ensembles of measured results rather than to any separated results. One can say that a coherent state of any quantum system causes another though any measured result does not cause any other one. However, this would require for the system in question to be absolutely separated from all the rest in the universe, which is impossible because of the phenomena of entanglement.

4. Any statistical ensemble, which obeys probabilistic causality, can be substituted by the isomorphic quantum one resulting in the same statistical ensemble after measurement.

\section{IF QUANTUM MECHANICS IS THE SOLUTION, WHAT SHOULD BE THE PROBLEM?} I have hinted the famous answer "42" as a metaphor of "The present situation in quantum mechanics", re-coining the title of a philosophical and critical review published by Schrödinger (1935) eighty years ago. The answer, i.e. quantum mechanics, is available, however the question, to which it should refer, is unknown yet and yet.

The present paper is an attempt to imagine or deduce the problem judging only by the answer rather than by the prejudice known as its interpretations. Perhaps, that intention would met sharp criticism for quantum mechanics is a tool working exceptionally well, on the one hand, and at least a few of the interpretations of quantum mechanics are accepted in the corpus of human knowledge, on the other hand. One can admit that the paper is skeptical to both and thus doubts 
them. This is not true. The text is skeptical only to that quantum mechanics and its interpretations are all truth rather than they are true.

This means that some crucial link is omitted in the picture of quantum mechanics so that its whole remains yet incomprehensible therefore imposing a functional and abstractly mathematical approach to quantum mechanics as to something, which works somehow... however works very well.

\section{TIME AS THE CRUCIAL LINK}

There is a natural prejudice about the course of time as an "arrow" from the past to the future. That prejudice implies the universal understanding of the future as the continuation of the past, and thus the principles of causality and entailment. Causality underlies almost all knowledge of mankind, and entailment: language, logic, and thinking.

Nevertheless the experience of time is quite different:

Time is, first of all, "now" when all happens and the human being makes choices. Those choices cannot change the past, but influence essentially the future. Neither the past nor the future are directly available in the experience of time, and rather reconstructed as memory and expectation.

Consequently that natural prejudice about the course of time is rather inconsistent to the experience of time as "now", and causality or entailment: to choice. This reflects in the problem of free will, on the one hand, and in the relation between the objective time of causality and entailment and the subjective time of choice and free will, on the other hand.

Another viewpoint to the same problem can offer a different understanding about the course of time closer to the experience of time: Time moves from the uncertain and unordered future to the well-ordered past by the choices made in the present. If one extrapolates the well-ordered past to the future, one would obtain the natural prejudice about the course of time, after which the future is expected to be similar to the past and thus ordered at least partly.

That time moving from the future to the past, as above, suggests that the past and the future are two absolutely different media in relation to ordering and thus to information as the quantity referable to ordering: The past is well-ordered even a mathematical sense while the future is absolutely unorderable even in principle. The collision between those two media generates the "thin line" of the present between them and thus the necessity of choices to conform both media to each other. Indeed a suitable series of choices can transform any collection in a well-ordered sequence of its elements. An analogical property is available in set theory as the equivalence of the axiom of choice and the well-ordering theorem.

Consequently, the experience of time should underlie the philosophical understanding of time, which in turn can reconstruct the natural prejudice about the course of time as a very important, but particular and borderline case of "no (or minimal) choice". Then the objective time should be 
interpreted also as a particular and borderline case of "neither choices nor free will" in relation to the subjective time of choices and free will.

That "subjective" viewpoint to time is shared at least by one experimental science such as quantum mechanics: Conway and Kochen $(2006$; 2009) deduce the "free will theorems" from its

principles: any item in the universe shares the "valuable commodity" of free will and choice if the experimenter possesses it.

\section{THE PROBLEM OF QUANTUM MECHANICS ACCORDING TO ITS HISTORY}

Any history of how quantum mechanics has appeared includes: Kelvin's metaphor about the two little clouds of the horizon of physics, Max Planck's solution of black-body radiation, Einstein's explanation of photoelectric effect, Niels Bohr's model of the atom, Louis de Broglie's waveparticle duality, Heisenberg's principle of uncertainty, matrix mechanics, wave mechanics, the equivalence of the last two, Max Born's probabilistic interpretation of quantum mechanics, the principle of complementarity, the principle of correspondence, quantum mechanics formulated in Hilbert space, etc.

However the collection of all those facts is often interpreted in a misleading way: The very small physical objects, the quantity of action of which is commeasurable with the Planck constant, are discrete. That discreteness is gradually transformed into the continuity of all quantities as to the physical bodies of our experience. The formalism of quantum mechanics based on Hilbert space represents this transformation. However it implies very ridiculous corollaries such as: both Born's (probabilistic) and Hugh Everett III's (many-worlds) interpretations, Einstein-Podolsky-Rosen's “paradox", Schrödinger's “cat”, Wigner's “friend" and many others, which should not be referred to reality by itself but to the mathematical model of quantum mechanics, which will be ostensibly perfected in future.

Consequently, the mathematical solution involved by quantum mechanics is ostensibly only partly relevant to its problem for the really relevant mathematical solution is forthcoming.

However, the expected relevant solution has not yet appeared, many of the ridiculous corollaries were ultimately confirmed experimentally and no one of them has be able to be refuted. Almost all physicists use just the standard mathematical formalism of quantum mechanics and escape to reflect on its metaphysical interpretations. So, the mathematical formalism seems to be the true unlike its philosophical interpretations being even redundant in a sense. The real solution is available, and the problem, to which it refers, appears to be unimportant and petty, but never mind. That is the situation in the philosophy of quantum mechanics, which can be designated by the metaphor " 42 ": The answer is clear, but what on earth is the question? 
V THE NECESSARY AND SUFFICIENT FACTS RELEVANT TO THE HIDDEN PROBLEM OF QUANTUM MECHANICS

One can attempt another approach to the real problem of quantum mechanics starting from its formalism and thus also from the ridiculous corollaries rather than from the presupposed interpretations of it. That approach would use the available mathematical formalism as the reliable base from which the true, but hidden yet problem of quantum mechanics might be deduced. This means that the mathematical formalism, i.e. the "answer", is equivalent to the problem, i.e. the "question", in a sense for both answer and question are mutually deducible from each other. This is admissible as the "ridiculous corollaries" are granted as true in that approach.

Furthermore, it hints the idea for the problem to be not only deduced, but to be deduced even axiomatically from the formalism as the formalism is just mathematical and thus easily representable axiomatically. Even more, those axiomatic features necessary and sufficient for both "answer and question" to be deduced from them can be embedded in the real facts of the history of quantum mechanics thus interpreting it. Those fundamental facts can be the following: (1) wave-particle duality; (2) the probabilistic (Born 1927) and many-worlds (Everett III 1957) interpretations of quantum mechanics; (3) Hilbert space as the basic mathematical formalism of quantum mechanics; (4) the "no hidden variables" theorems in quantum mechanics. The concept of (5) quantum information and the (6) Schrödinger equation linking these properties (1-4) of the formalism of quantum mechanics to its utilization and meaning will be considered a little latter and in detail.

3.1. Wave-particle duality addresses the dual behavior of any quantum object such as an atom, an electron, a photon, etc. according to the kind of experiment: One and the same thing can be considered both as a particle and as a wave. However this is not contradictory because it behaviors either as a particle or as a wave in any given experiment just according to its kind. One can say that being either a particle or a wave is an accidental feature depending only on the external circumstances rather than an essential property referring to its nature and definition.

Wave-particle duality should be reformulated as the invariance of the physical laws to all discrete and smooth (being necessary continuous) motions in the usual three-dimensional Euclidean space. Thus, the duality can be considered as a generalization of Einstein (1918)'s equivalence in general relativity to all reference frames corresponding to the smooth motions. Then waveparticle duality means for the Einstein equivalence to be extended including also all discrete motions, i.e. all quantum leaps.

3.2. Hilbert space as the basic mathematical formalism of quantum mechanics has been introduced after the merging of matrix and wave mechanics ultimately completed in John von Neumann's "Mathematical foundations of quantum mechanics" (1932): Any state of any quantum system can be represented by a wave function, which is a "point" in Hilbert space, and 
vice versa. Any physical quantity is a kind of linear operator in Hilbert space (a "hyper-maximal operator" according to Neumann's terminology).

Hilbert space is the simplest mathematical structure, the "points" of which can represent equally well a leap and a smooth motion in the three-dimensional Euclidean space and thus are invariant both to discrete and to smooth morphisms of it therefore satisfying the generalized Einstein principle above.

3.3. The probabilistic (Born 1957) and many-worlds (Everett III 1957) interpretations of quantum mechanics connect the wave function with the statistical ensemble of observed results after measuring the quantum state possessing this wave function. The square of the module of the wave function corresponds unambiguously to the probability for the state to be measured according to the Max Born interpretation. Any state whether the observed or other can be considered as an actual member of a statistical ensemble of all states and thus as a "world" continuing to exist in parallel to ours according to the "many-worlds" interpretation. The measurement or observation, which has chosen somehow to be in our world, therefore has called the splitting between them at the same time.

Though the concept of wave function as a point in Hilbert space has been initially involved to satisfy the uniform description of quantum leaps and smooth motions (or "wave-particle duality"), it turns out to have additionally another symmetry or invariance after both probabilistic and many-world interpretations: It is able to describe uniformly a coherent state (which is unorderable in principle for the "no-hidden variables" theorems) and the corresponding statistical ensemble (which is always well-orderable). Thus, it involves necessarily: (1) the well-ordering theorem equivalent to the axiom of choice, in the mathematical model; and (2) furthermore, choice and information as the quantity of choices, in quantum mechanics. The same can be interpreted: (1) ontologically as the invariance of the possible and actual, (2) mathematically as a kind of set-theory invariance in relation to the axiom of choice, and (3) as an invariance embodied in the concept of information.

Nevertheless, the link between wave-particle duality and the newly formulated "possibilityactuality duality" in quantum mechanics needs some explanation for it should be not an accidental feature infused by the mathematical model but an essential part of the problem, which is researched and re-searched.

3.4. The meant "no hidden variables" theorems in quantum mechanics are that of John von Neumann (1932: 157-173) and that of Kochen and Specker (1968), which can be considered both as an amplification and as a generalization of the former. They show that the "unused half" of variables in comparison to classical mechanics for a mechanical system to be exhaustedly described is redundant in principle if the mathematical model is that of Hilbert space. They can be also considered as a corollary from the above statements (3.1-3.3) and thus are able not to be included within the corpus of necessary axioms. However, they are rather useful for the 
mentioned two dualities or invariances to be unified into a single one: Indeed any of both dualities "spares" the one half of variables as "dual" and thus "redundant":

In other words, classical mechanics accepts that a leap and a smooth motion are different from each other: therefore each of them needs a proper description. Quantum mechanics involves their invariance and thus "spares" the one description. Classical mechanics accepts that a possible state and an actual one are different. So, each of them needs a relevant description. Analogically, quantum mechanics introduces their equivalence and thus goes without the one description. Consequently, that "economy" is the common base shared by both dualities. The essence of that "economy" is information, especially quantum information. Their units, correspondingly a bit and a qubit, are the "currency" of the "saving".

\section{THE QUANTUM INFORMATION}

The concept of quantum information introduced by quantum mechanics may be used to describe uniformly future and past and thus the present as the frontier of time.

The conception of quantum information was introduced in the theory of quantum information studying the phenomena of entanglement in quantum mechanics. The entanglement was theoretically forecast in the famous paper of Einstein, Podolsky, and Rosen (1935) and independently by Shrödinger (1935) deducing it from Hilbert space. However, the former three demonstrated the forecast phenomenon as the proof of the alleged "incompleteness of quantum mechanics". John Bell (1964) deduced a sufficient condition as an experimentally verifiable criterion in order to distinguish classical from quantum correlation (entanglement). Aspect, Grangier, and Roger (1981, 1982) confirmed experimentally the existence of quantum correlations exceeding the upper limit of the possible classical correlations. The theory of quantum information has thrived since the end of the last century in the areas of quantum computer, quantum communication, and quantum cryptography.

The fundament of quantum information is the concept of 'quantum bit', "qubit" definable as the normed superposition of any two orthogonal subspaces of complex Hilbert space as follows: 'Qubit' is: $\alpha|0\rangle+\beta|1\rangle$ where $\alpha, \beta$ are complex numbers such that $|\alpha|^{2}+|\beta|^{2}=1$, and $|0\rangle,|1\rangle$ are any two orthonormal vectors (e.g. the orthonormal bases of any two subspaces) in any vector space (e.g. Hilbert space, Euclidean space, etc.). Thus Hilbert space underlying quantum mechanics is representable as the quantity of quantum information and any wave function, i.e. any state of any quantum system being a point in it can be seen as a value of that quantity. Consequently, all physical processes turn out to be quantum-informational, and nature or the universe may be interpreted as a quantum computer processing quantum information.

The qubit is also isomorphic to a ball in Euclidean space, in which two points are chosen: the one chosen within the ball, and the other being the orthogonal projection on its surface, i.e. as a mapping of a unit ball onto its surface (or any other unit sphere). 
Quantum information can be interpreted as the transfinite generalization of information. Its unit, a qubit, can be interpreted as a choice among an infinite set of alternatives. It generalizes the unit of classical information, a bit, which refer to a finite set of alternatives.

If one uses the notion of "length of now" defined as the period of the de Broglie (1925) wave associable to any physical item, the "length of now" of the apparatus can be visualized as a random chosen point onto the segment of the "length of now" of the measured quantum entity. Thus quantum mechanics is forced to describe uniformly the future, present and past of the investigated system. Future is represented as a coherent whole, present as a choice among infinitely many alternatives, and past as a well-ordering obtained as a result of a series of choices. The concept of quantum information unifies the future, present, and past as a measurable physical quantity. It describes the frontier of time, that "now", which transforms future into past.

The theorems about the absence of hidden variables in quantum mechanics (Neumann 1932; Kochen, Specker 1968) demonstrate that the mathematical formalism of quantum mechanics implies that no well-ordering of any coherent state might exist before measurement. However, the same coherent state is transformed into a well-ordered series of results in time after measurement. In order to be equated the state before and after measurement, the well-ordering theorem equivalent to the axiom of choice is necessary. The measurement mediating between them should be interpreted as an absolutely random choice of an element of the coherent state, for which no constructive way (equivalent to some "hidden variable") can exist in principle. The quantity of quantum information can describe uniformly the state before and after measurement (equivalent to a choice among an infinite set). Any wave function, being a given value of quantum information, "bounds" an unorderable and a well-ordered state as the quantity of qubits (i.e. the "infinite choices") necessary for the latter to be obtained from the former.

The quantity of quantum information is also the ordinal number corresponding to the infinity series of finite choices. Both definitions of 'ordinal' (Cantor 1897; Neumann 1923) are applicable as the ordinals are relatively "small". The ordinal number defined in Cantor - Russell (Whitehead, Russell [any edition]: Vol. 2(*153), Vol. 3(*251)) generates a statistical ensemble while that in Neumann, a well-ordering. Both correspond one-to-one to a coherent state as the one and same quantity of quantum information containing in it. One can accept that both definitions of "ordinal" are equivalent to where both definitions are applicable, i.e. to relatively "small" transfinite ordinals. Furthermore, that equivalence in turn corresponds to the equivalence of both definitions of the quantity of information: (1) as mutual entropy, i.e. as the functional of some probability distribution on the basis of another; (2) as the minimal length of an algorithm, which is able to reorder a well-ordering into another, i.e. the transfinite generalization of the Kolmogorov $(1965 ; 1968)$ definition of information. In fact, the concept of quantum information rests on the latter equivalence therefore corresponding thus to the equivalence of the ways for "ordinal" to be defined. Even more, one can offer the hypothesis that quantum information and 
thus the equivalence of the well-ordered and "statistical-ensemble" ordinals underlies the fundamental equation of quantum mechanics: the Schrödinger equation, therefore elucidating its meaning.

The concept of quantum information allows of a large-scale synthesis: It links the physical processes studied by quantum mechanics to the theory of information, and by meditation of it, both to set theory and to the ontology of time in philosophy. The core of that synthesis is just "quantum information", which explains the course of time as ordering and relations of orderings (i.e. in terms of information)

\section{THE SCHRÖDINGER EQUATION}

We suggest that the Schrödinger equation is well-known in all modifications. So, it will be sufficient and even more suitable to be described only generally in the non-relativistic case for a single "particle":

$$
i \hbar \frac{\partial \Psi(\boldsymbol{r}, t)}{\partial t}=\left[-\frac{\hbar^{2}}{2 \mu} \Delta+V(\boldsymbol{r}, t)\right] \Psi(\boldsymbol{r}, t)
$$

Here:

$\checkmark$ " $i$ " means imaginary unit: $i^{2}=-1$

$\checkmark$ “ $\hbar$ " means the Planck constant per unit of angle: $\hbar=\frac{h}{2 \pi}$

$\checkmark$ " $\Psi(\boldsymbol{r}, t)$ " is the wave function of space radius-vector $\boldsymbol{r}=\boldsymbol{r}(x, y, z)$, and time $\mathrm{t}$

$\checkmark$ " $\mu$ " is reduced mass allowing of $\mathrm{n}$ "particles" to be considered as a single one:

$$
\frac{1}{\mu}=\sum_{i=1}^{n} \frac{1}{m_{i}}
$$

$\checkmark$ " $\Delta$ " is the Laplacian, a differential operator: $\Delta=\nabla^{2}=\frac{\partial}{\partial x^{2}}+\frac{d}{d y^{2}}+\frac{d}{d z^{2}}$

$\checkmark$ " $V(\boldsymbol{r}, t)$ " is potential energy, and the expression " $-\frac{\hbar^{2}}{2 \mu} \Psi(\boldsymbol{r}, t)$ " corresponds to kinetic energy

This equates the first time derivative (therefore the change in time) of the wave function (therefore a value of quantum information) of any quantum system to the algebraic sum of (1, with sign minus sign) the second space derivative (laplacian and thus the tensor product of two changes in space) of the same wave function (and the same value of quantum information) corresponding to kinetic energy in classical mechanics and of (2, with a plus sign) the product of a potential function and the same wave function, corresponding to potential energy in classical mechanics. Both sides of the equation have the physical dimension of energy. The coefficients involved in the equation are the Planck constant, the mass of the system (more exactly, the reduced mass of the system), and the imaginary unit, $i$, which is very important for the information interpretation of the equation. 
A few preliminary comments are necessary:

5.1. The Schrödinger equation is a generalization of the fundamental law of energy conservation in classical mechanics: Indeed the former implies the latter if the wave function is a constant as a particular case. In turn, the generalization involves just complex functions being inapplicable as to any real functions. One may suggests then that quantum information corresponds to a relevant generalization of energy already definable as to complex values therefore involving a second, i.e. just imaginary dimension of energy. That dimension is interpretable as "time energy" unlike kinetic and potential energy both being callable "space energies". Unfortunately the suitable term of "space-time energy" as equivalent to quantum information would call a series of messes for special and general relativity. Another option for them is to be designated correspondingly as temporal and spatial energy. Then, one of the possible meanings of the Schrödinger equation would be their equating. Furthermore the spatial energy would correspond to quantum information defined as a statistical ensemble (or as an ordinal in Cantor - Russell), and the temporal energy, defined as a transfinite ordinal (in Neumann).

5.2. The potential and kinetic energy are clearly distinguished from each other in classical mechanics: Kinetic energy corresponds to the real motion depending only on its velocity, and potential energy describes the force in each spatial point acting on a tentative reference unit (e.g. a body or a material point) depending only on its position. The law of energy conservation means the way for the acting of the force to be transformed into the motion of some real item. The Schrödinger equation generalizes the classical understanding as follows:

The wave function corresponds to space in a different way in the cases of kinetic and potential energy: The tensor product of two identical and complementary (or "conjugate") wave functions is mapped into space if the energy is kinetic, and a single wave function is mapped into space in the case of potential energy. Indeed the latter can be interpreted as the action on an energymomentum unit thus depending only on the spatial coordinates. However this representation closer to the ideas of classical mechanics is "paraphrased" at present (and particularly in the Standard model) as a few kinds of fundamental symmetries: any symmetry is a relation between a set of symmetric elements as different and the same set as a single element being a class of equivalence to the symmetry in question. Thus, the concept of symmetry expresses differently but equivalently the underlying idea of quantum information: the choice of an element among a set of equivalent, but different alternatives. Nevertheless the form of the Schrödinger equation is universal referring to all possible symmetries rather than to the ones really observed in experiments until now or to the ones included in the Standard model.

5.3. The invariance of discrete leaps and smooth motions allows of the Schrödinger equation to be represented by the corresponding units: bits and qubits. Furthermore, one can introduce a new distinction between real (or kinetic) and potential qubits: the formers for kinetic energy, the 
latters for potential energy. Then, the Schrödinger equation can be formulated so: The energy of a "(trans)finite bit" is equal to the subtraction of the energy of a potential and a kinetic qubit. The necessary equivalence of potential and kinetic qubits is implied by the equivalence of the probabilistic and many-worlds interpretation ( 3.3 above) and in turn implies the equivalence of a bit of a transfinite position ("transfinite bit") and a bit of a finite position ("finite bit").

5.4. The Kochen - Specker (1968) theorem helps us to avoid a mess: the equation of the energies as above does not mean that a qubit can be equivalent to a bit, which would contradict to the direct corollary (Kochen, Specker 1968: 70), which is immediately after the main theorem of their paper (ibid., a few lines above). In fact the concept of energy conservation (only generalized in the Schrödinger equation), as Emmy Noether's (1918) fundamental theorem shows, implies the equality of all moments of time. Consequently, the equation of energies implies that the past moment corresponding to a bit, the present moment: to a kinetic qubit, and the future moment: to a potential qubit are already presupposed as equivalent, but this does not refer to the bits and qubits themselves: They are fundamentally different, and only the quantity of their corresponding energy can be equated.

Summarizing all consideration above, one can suggest the following meaning of the Schrödinger equation in terms of quantum information: It generalizes energy conservation to past, present, and future moments of time rather than only to present and future moments as this does the analogical law in classical mechanics. Thus, it is the universal law of how "time flows" or in other words, about the course of time. Furthermore, resolving its problem, the Schrödinger equation suggests the proportionality (or even equality if the units are relevantly chosen) of the quantities of both quantum and classical information and energy therefore being a (quantum) information analog of Einstein's famous equality of mass and energy (" $\mathrm{E}=\mathrm{mc}^{2 "}$ ).

VIII THE PROBLEM IF QUANTUM MECHANICS IS ITS SOLUTION:

What is the universal law describing the course of time in any physical change therefore including any mechanical motion?

One can show quite shortly that the premises $(3.1,3.2,3.3,3.4 ; 4$, and 5$)$, from which it is deduced, are implied in turn from the so-formulated problem in accordance to the postulated equivalence of the problem and solution.

6.1 (for 3.1): The leap from past to future is equivalent to the smooth transition from present to future: This implies the invariance meant in 3.1.

6.2 (for 3.2): Hilbert space is the simplest mathematical structure unifying the discrete (arithmetic) and smooth (geometry) necessary for 6.1 and thus for 3.1.

6.3 (for 3.3): The energetic equivalence of the future, the present, and the past for Schrödinger equation implies an analogical energetic equivalence of possibilities (probabilities), actualities (many-worlds) and even of well-orderings (logics). (The latter is neither presupposed nor discussed.) 
6.4 (for 3.4): The uniform description of the fundamentally unorderable future and wellordered past implies the missing of hidden variables.

6.5 (for 4): The quantity of information and quantum information as a generalization of the former allows for the three modes of time to be described uniformly as the three successive phases of any choice: the coherent state of all options for the future; the actual action of choice of an option for the present; and the well-ordering as the result of a series of choices for the past.

6.6 (for 5): All considerations imply that the meaning of the Schrödinger equation refers to the course of time.

\section{CONCLUSIONS ABOUT OCCASIONALISM IN QUANTUM MECHANICS}

Any conception of causality including that of occasionalism was intentionally omitted in the discussion after it had been involved only in Introduction. This was necessary for any interpretation not to bring in inferring the real but hidden problem of quantum mechanics just according to its mathematical formalism. Once the meaning of the Schrödinger equation has been already deduced, one can conclude a few interpretations from it:

1. The Schrödinger equation describes the reversed course of time in comparison to that suggested in the theories of causality. Furthermore, it describes quantitatively that reversed course of time. On the contrary, any theory of causality presupposing the standard "arrow of time" explains it only qualitatively as a rigorous sequence, i.e. as a well-ordering necessarily starting in some past moment. One is not able to deduce any quantitatively law about the course of time in the patter case for it reconstructs the course of time secondarily and approximately,

2. The usual theories of causality in quantum mechanics, physics, and exact sciences at all are intimately linked to energy conservation, which was here elucidated as the absolute equation of potential and kinetic energy, thus entirely neglecting the left side of the Schrödinger equation or equating it constantly to zero therefore requiring for the wave function to be constant in all spacetime. That constancy of the wave function is inconsistent not only with quantum mechanics grounded on its variability, but even with the following obvious fact in classical mechanics: The existence of any particle or body is limited to a closed space-time area where the probability to be measured or observed is 1 , and out of which is 0 . Therefore the wave function is not able to be constant in all space-time even in classical mechanics. That impossibility being a kind of "reductio ad absurdum" implies the violation and thus the necessity of generalization of energy conservation what the Schrödinger equation is.

3. Energy conservation equates all moments of time. This is not only any empirical or experimental fact, but even it is inconsistent with the human experience for a moment in the past, that in the present and that in future are apparently different in quality. Indeed there is no way to compare empirically or experimentally the length of a present moment, and that of another present moment, or with those of a past or future moment. If those lengths are different, action 
conservation rather than energy conservation will hold as though general relativity and statistic thermodynamics seems to state in fact.

4. Nevertheless that action conservation is not an enough generalization of energy conservation as to quantum mechanics for it equates quantitatively future, present and past moments in the Schrödinger equation. This implies information conservation in a sense, of which action conservation is a particular case, including furthermore the mutual transition and transformation between information (entropy, probabilities) and the physical quantity of action. Those transition and transformation can be interpreted philosophically as the transition and transformation of the ideal and material.

5. Quantum occasionalism demonstrates even quantitatively by means of the Schrödinger equation that future is what "causes" the present, and the latter in turn is what "causes" the past. However that kind of "reverse causality" needs free choice in the present to be realized unlike classical determinism.

6. Quantum occasionalism considers and equates three forms of information therefore unifying information theory and mechanics, and thus even mathematics and physics. Those three form of information turn out to be:

$\checkmark$ Transfinite strings of binary cells (a generalization of Kolmogorov's definition of information)

$\checkmark$ Quantum information in the form three-dimensional unit balls (ensembles of fermion statistics), and at last:

$\checkmark$ Quantum information in the form of characteristic function of some probability distribution (ensembles of boson statistics)

7. Those three forms of information correspond to the past, present, and future, and the essence of the Schrödinger equation is just they to be equated to each other. 


\section{REFERENCES:}

Aspect, A., Grangier, R., Roger, G. (1981) "Experimental Tests of Realistic Local Theories via Bell's Theorem," Physical Review Letters 47 (7): 460-463.

Aspect, A., Grangier, R., Roger, G. (1982) "Experimental Realization of Einstein-Podolsky-RosenBohm Gedanken Experiment: A New Violation of Bell's Inequalities," Physical Review Letters 49 (2): 91-94.

Bell, J. (1964) "On the Einstein - Podolsky - Rosen paradox," Physics (New York) 1 (3): 195-200.

Born, M. (1927) "Physical aspects of quantum mechanics," Nature 119: 354-357.

Broglie, L. de (1925) "Recherches sur la théorie des quanta" (Researches on the quantum theory), Thesis (Paris), 1924. Annales de Physique (Paris, 10-ème série) 3: 22-128.

Cantor, G. (1897) "Beitrage zur Begrundung der transfiniten Mengenlehre (Zweiter Artikel)," Mathematische Annalen 49 (2): 207-246.

Conway, J., Kochen, S. (2006) "The Free Will Theorem," Foundations of Physics 36 (10): 1441-1473.

Conway, J., Kochen, S. (2009) "The Strong Free Will Theorem," Notices of the AMS 56 (2): 226-232.

Einstein, A. (1918) "Prinziplelles zur allgemeinen Relativitätstheorie," Annalen der Physik 55 (4): 241-244.

Einstein, A., Podolsky, B., Rosen, N. (1935) "Can Quantum-Mechanical Description of Physical Reality Be Considered Complete?" Physical Review 47 (10): 777-780.

Everett III, H. (1957) „Relative state” Formulation of Quantum Mechanics,” Reviews of Modern Physics 29 (3): 454-462.

Kochen, S., Specker, E. (1968) “The problem of hidden variables in quantum mechanics," Journal of Mathematics and Mechanics 17 (1): 59-87.

Kolmogorov, A. (1965) "Tri podkhoda k opredeleniyu "kolichestvo informatsii" (Three approaches to the definition of the quantity of information), Probl. Inf. Transm. 1: 3-11.

Kolmogorov, A. (1968) "Logical basis for information theory and probability theory," IEEE T. Inform. Theory IT-14: 662-664.

Neumann, J. von (1923) "Zur Einführung der trasfiniten Zahlen," Acta litterarum ac scientiarum Ragiae Universitatis Hungaricae Francisco-Josephinae, Sectio scientiarum mathematicarum, 1 (4): 199208.

Neumann, J. von (1932) Mathematische Grundlagen der Quantenmechanik, Berlin, Springer, pp. 167-173 (Chapter IV.2).

Noether, E. (1918) "Invariante Variationsprobleme," Nachr. d. König. Gesellsch. d. Wiss. zu Göttingen, Math-phys. Klasse 1918, pp. 235-257.

Schrödinger, E. (1935) "Die gegenwärtige situation in der Quantenmechanik," Die Naturwissenschaften 23 (48): 807-812; 23 (49): 823-828; 23 (50): 844-849.

Whitehead, A. N., Russell, B. (any edition). Principia Mathematica, Vol. 2(*153), Vol. 3(*251). 